\title{
Comparison of a Histoplasma/Blastomyces Lateral Flow Assay to Enzyme Immunoassay Using Dog and Cat Sera
}

Janelle Renschler, Logan Cheney, Amanda Albers, Michelle Durkin and L. Joseph Wheat

MiraVista Diagnostics, Indianapolis, Indiana, USA

\section{INTRODUCTION}

- Quantitative enzyme immunoassays (EIAs) for Histoplasma and Blastomyces antigen are highly sensitive for the detection of fungal antigen to aid in the diagnosis of histoplasmosis and blastomycosis in dogs and cats. ${ }^{1-3}$ These assays show nearly complete cross reactivity; therefore, both infections may be detected by a single antigen test.

- A serum lateral flow assay (LFA) was developed for human histoplasmosis, and this might be used in dogs and cats.

- Use of the LFA as a point-of-care assay in veterinary clinics would allow for more rapid diagnosis and earlier treatment of systemic mycoses

- The objective of this study was to compare agreement between the Histoplasma antigen LFA and the quantitative EIAs for Histoplasma and Blastomyces, using canine and feline serum.

\section{METHODS}

- Serum samples previously tested in either EIA, each tested in duplicate by LFA

- 11 EIA-negative (22 LFA devices)

- 21 ElA-positive (42 devices)

- Ranged from $0.2 \mathrm{ng} / \mathrm{mL}$ to $19 \mathrm{ng} / \mathrm{mL}$

- Species distribution: 12 feline, 20 canine

- Serum was treated with EDTA and extracted supernatant was loaded onto the LFA device

- LFA devices were interpreted:

- Visually by three qualified human evaluators (Fig. 1)

- Two automated readers (Cube-Reader, opTricon $\mathrm{GmbH}$, Germany) (Fig. 2).

- A consensus RANN score (ranging from 0-10 based on intensity of the test line compared to scorecard standards) was obtained by using two evaluator agreement. The samples were randomized and evaluators were blinded to EIA results.

- Cube reader $T_{\text {mean }}$ also recorded and reported as positive or negative based on cutoff calculation

\section{RESULTS}

- Results comparison summarized in Table 1

- Kappa agreement with EIA:

\section{- Visual assessment $\mathbf{0 . 8 5 8}$}

- Automated readers $\mathbf{0 . 8 6 1}$ and $\mathbf{0 . 7 6 0}$

- ElA-negative samples with positive LFA results included:

- Three devices with very low consensus RANN scores (1-2)

- Two were also positive by both cube readers

- One was positive by only one cube reader

- All were canine samples

- EIA-positive samples with negative LFA results included:

- One device with very low positive EIA result $(0.5 \mathrm{ng} / \mathrm{mL})$ and negative results by all LFA evaluations

- Three additional devices with very low positive EIA results, negative by one cube reader

- All were feline samples

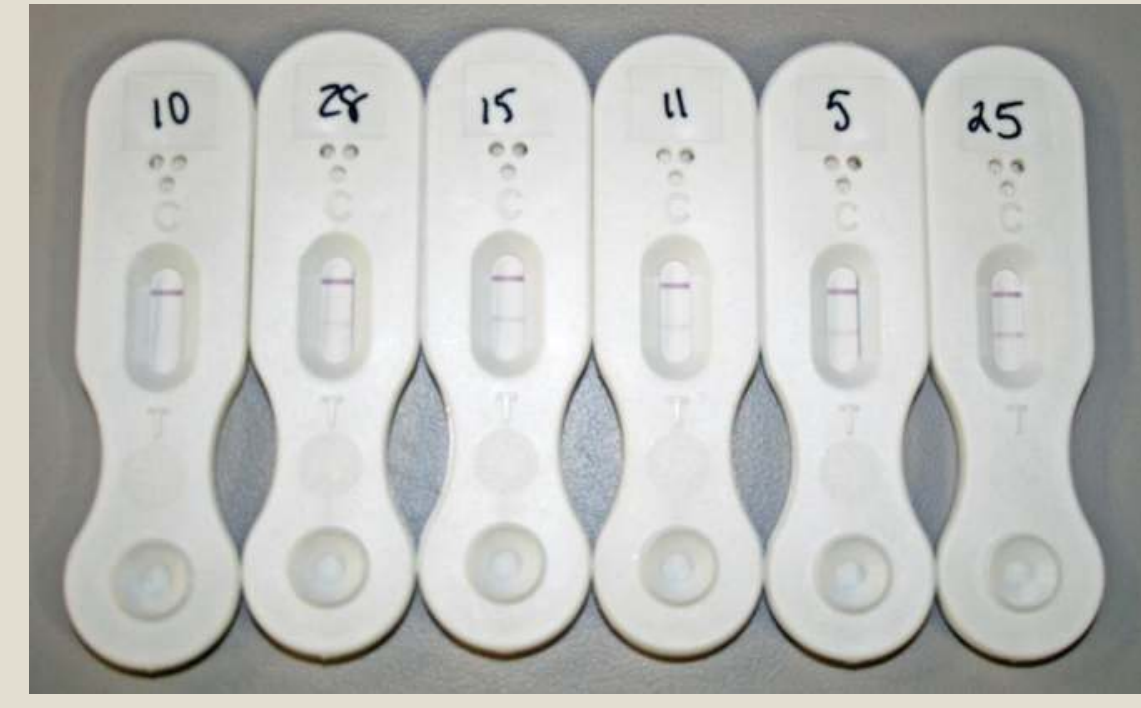

Fig. 1. LFA devices with RANN scores ranging from negative to high positive

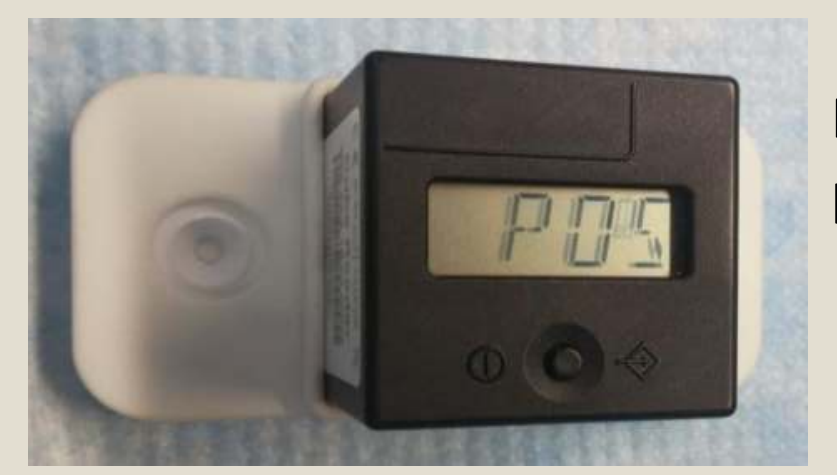

Fig. 2. opTricon Cube-Reader placed over the LFA device

Table 1. Comparison of Histoplasma or Blastomyces EIA results with LFA by visual assessment or opTricon Cube-Readers

\begin{tabular}{|c|c|c|c|c|c|c|}
$\begin{array}{c}\text { EIA } \\
\text { Result }\end{array}$ & \multicolumn{2}{c|}{$\begin{array}{c}\text { LFA Visual } \\
\text { Result }\end{array}$} & \multicolumn{2}{|c|}{$\begin{array}{c}\text { LFA Cube 1 } \\
\text { Result }\end{array}$} & \multicolumn{2}{|c|}{$\begin{array}{c}\text { LFA Cube 2 } \\
\text { Result }\end{array}$} \\
\hline & - & + & - & + & - & + \\
\hline- & 19 & 3 & 20 & 2 & 19 & 3 \\
\hline+ & 1 & 41 & 2 & 40 & 4 & 38 \\
\hline
\end{tabular}

\section{CONCLUSIONS}

\section{- Good agreement between LFA and EIA}

- Uncommon false positive LFA results from some canine samples; very faint bands

- also detected by automated readers (not evaluator error)

- Rare false negative LFA results from samples with low antigen concentration

- Visual assessment outperformed automated readers

- LFA may be a useful diagnostic tool for rapid serum diagnosis of histoplasmosis or blastomycosis in dogs and cats

\section{MiraVista II DIAGNOSTICS}

